\title{
CML—where do we stand in 2015?
}

\author{
Rüdiger Hehlmann
}

Received: 28 January 2015 / Accepted: 28 January 2015

(C) Springer-Verlag Berlin Heidelberg 2015

\section{Introduction}

The progress with CML treatment continues at a rapid pace. Five-year survival ranges around $91 \%$ and 10-year survival around $84 \%$ up from $11 \%$ with busulfan 30 years ago. Figure 1 gives an overview of what has been achieved over the past 32 years.

Table 1 summarizes published evidence on 5-10-year survival as reported from ten selected randomized and observational studies. Four studies report 8-10-year survival rates after 5.8-9.9 years of median observation time.

Deep molecular responses are achieved by the majority of imatinib-treated patients. It is expected that second-generation tyrosine kinase inhibitors (TKI) will induce even higher response rates earlier. Treatment goal for CML is no longer palliation or prolongation of survival, but discontinuation of therapy and cure.

This special CML issue of the Annals summarizes the situation in CML in 2015 through 15 state-of-the-art reviews on various aspects of research, management, and public health. As the basis for progress with diagnosis and treatment, review 1 updates the molecular biology of CML, the role of BCR$\mathrm{ABL}$ in proliferation and progression, and important pathways altered in CML.

Reviews 2, 3, and 4 summarize current choices of first- and second-line treatments including their advantages and pitfalls, discuss causes of resistance, and give management recommendations according to predefined cytogenetic and molecular response levels. Adverse drug effects of the various TKI may limit indications of the newer TKI. Review 5, therefore, advises which adverse effects should be expected, how

R. Hehlmann $(\bowtie)$

Medizinische Fakultät Mannheim, Universität Heidelberg,

Pettenkoferstr. 22, Mannheim 68169, Germany

e-mail: R.Hehlmann@urz.uni-heidelberg.de adverse effects can be avoided, and how they can be managed once they do occur. Management of CML blast crisis is a challenge to hematologists also in the TKI era, although blast crisis occurs at a much lower rate than in the pre-TKI era and mostly early after the start of treatment (review 6). Management of CML and pregnancy gains increasing importance with improved long-term survival, and review 7 addresses the safety of TKI during pregnancy, treatment alternatives, and fertility preservation.

Hematopoietic cell transplantation continues to be an option in CML, not only in late disease phases but also in early good risk patients with a low transplantation risk (review 8).

Review 9 addresses the current experience with TKI discontinuation and what the open questions are in order to improve the rate of potential cures. An option to improve outcome of TKI treatment may be interferon $\alpha$ either in combination with TKI or as maintenance after TKI discontinuation (review 10). Since prognosis of CML changes according to type of therapy, a new prognostic score has been proposed for TKI-treated patients. Due to the low rate of CML-related deaths, other causes of death may compete and require consideration (review 11).

Disease monitoring has proved to be the key factor for successful disease management. Failure to achieve complete cytogenetic response has remained the most important indication for a change of therapy. Molecular monitoring is increasingly important as an early indicator of failure and as an early response predictor and criterion for treatment discontinuation. Interlaboratory standardization has successfully been achieved by a concerted internationally exemplary European effort (review 12). Chances and limitations of early response predictors of survival are analyzed in review 13.

Due to the reduced mortality, prevalence of CML is increasing worldwide (review 14) which poses serious health economic challenges due to high TKI costs. Review 15 deals with the cost of care as a public health issue with an eye on 


\section{Survival with CML over time}

\section{The German CML-Study Group experience 9/2014}

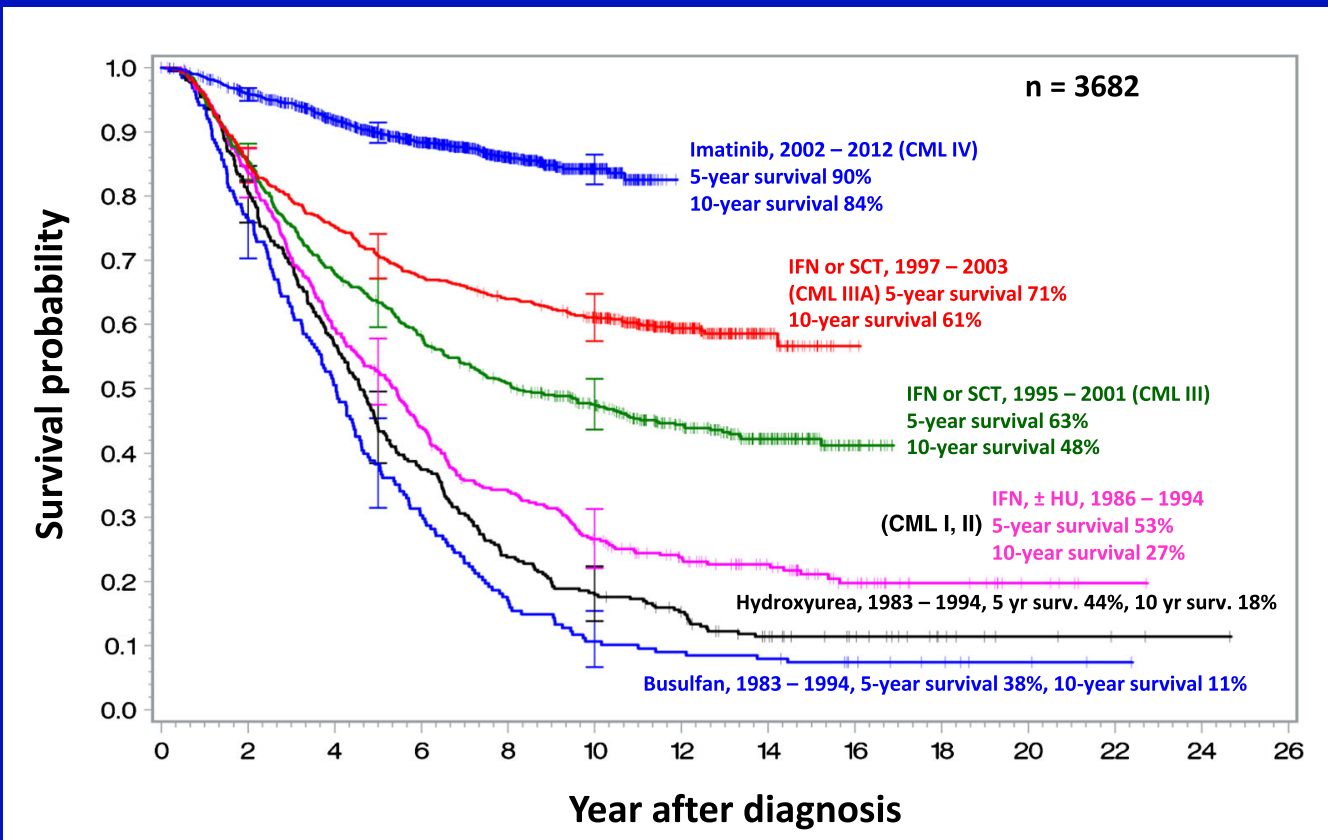

German CML Study Group, update 2014

Fig. 1 Survival with CML as observed in five consecutive randomized treatment optimization studies of the German CML Study Group 1983-2014

Table 1 Long-term survival data of CML 2014

\begin{tabular}{|c|c|c|c|c|c|c|}
\hline Study & Treatment mg & $n$ & $\begin{array}{l}\text { Age at diagnosis, } \\
\text { median, years }\end{array}$ & $\begin{array}{l}\text { 5-year survival } \\
(\%)\end{array}$ & $\begin{array}{l}\text { 10-year survival } \\
(\%)\end{array}$ & $\begin{array}{l}\text { Med. observation } \\
\text { time (years) }\end{array}$ \\
\hline CML-IV [1] & IM 400-800 & 1536 & 53 & 90 & 84 & $7.1(\max .11 .7)$ \\
\hline IRIS [2] & IM 400 & 553 & 50 & 89 & 85 (8 years) & 8 \\
\hline GIMEMA [3] & IM 400-800 & 559 & 52 & 90 & NA & 5 \\
\hline Hammersmith [4] & IM 400 & 204 & 46.3 & 83 & NA & 3.2 \\
\hline PETHEMA [5] & IM 400 & 210 & 44 & 97.5 & NA & 4.2 \\
\hline \multirow[t]{2}{*}{ TOPS [6] } & IM 400 & 157 & 45 & 94 (4 years) & \multirow[t]{2}{*}{ NA } & 3.5 \\
\hline & IM 800 & 319 & 48 & 93.4 (4 years) & & 3.5 \\
\hline \multirow[t]{2}{*}{ MDACC [7] } & IM 400 & 70 & \multirow[t]{2}{*}{48.3} & \multirow[t]{2}{*}{ NR } & 80 & \multirow[t]{2}{*}{9.9 (min. 8) } \\
\hline & IM 800 & 201 & & & 84 & \\
\hline ILTE [8] (CCR only) & IM NR & 832 & $51^{\mathrm{a}}$ & 98 (6 years) & 95 (8 years) & 5.8 \\
\hline \multirow[t]{3}{*}{ ENEStnd [9] } & IM 400 & 283 & 46 & 92 & \multirow[t]{3}{*}{ NA } & \multirow[t]{3}{*}{5} \\
\hline & Nilo 600 & 282 & 47 & 94 & & \\
\hline & Nilo 800 & 281 & 47 & 96 & & \\
\hline \multirow[t]{2}{*}{ Dasision [10] } & IM 400 & 260 & 49 & 90 & \multirow[t]{2}{*}{ NA } & \multirow[t]{2}{*}{5} \\
\hline & Dasa 100 & 259 & 46 & 91 & & \\
\hline Median (estimate) & & & & 91 & 84 & \\
\hline
\end{tabular}

$N R$ not reported, min. minimum, IM imatinib, $N A$ not available, Nilo nilotinib, Dasa dasatinib

${ }^{a}$ At registration 
generic imatinib which will become generally available also in the EU and the USA in 2016.

The reviews of this issue cannot cover the rapidly evolving CML field completely, but they address issues of relevance for CML per se and for CML as a model disease. Solutions found for CML might be applied to other cancers in the future. The reviews provide a basis to better understand and critically follow ongoing developments in this expanding field in research, disease management, and promotion of public health.

Conflict of interest The author declares that he has no conflict of interest.

\section{References}

1. Hehlmann et al (2014) J Clin Oncol 32(5):415-23

2. Deininger et al ASH (2009) Abstract. 1126

3. Baccarani et al EHA (2009) Haematologica 94(suppl.2):254 Abstr. 626

4. de Lavallade et al (2008) J Clin Oncol 26(20):3358-63

5. Cervantes et al (2010) Haematologica 95(8):1317-24

6. Baccarani et al (2014) Int J Hematol 99(5):616-24

7. Sasaki et al ASCO (2014) Abstract 7024

8. Gambacorti-Passerini et al (2011) J Natl Cancer Inst 103(7):553-61

9. Larson et al ASCO (2014) Abstract 7073

10. Cortes et al ASH (2014) Abstract 152 